\title{
A molecular theory of large-solute diffusion
}

\author{
A. Yoshimori* \\ Department of Physics, Kyushu University, Fukuoka, 812-8581, Japan
}

Received September 4, 2007, in final form October 11, 2007

\begin{abstract}
The limit of a large solute in the molecular theory of diffusion developed by Yamaguchi et al. [Yamaguchi T. et al., J. Chem. Phys., 2005, 123, 034504] is studied. By the limit, the Stokes approximation to the hydrodynamic equations is derived in the outside region of a diffusing solute. The limit of a large solute also leads to equations in the inside region of the solute. The analytical solution of the inside equation allows one to derive the boundary condition, which is needed on the surface of the solute when the hydrodynamic equations are calculated. The boundary condition includes stick and slip boundary conditions employed by the Stokes law, in the special case. Besides stick and slip conditions, other conditions can be expressed. The boundary condition depends on properties of a solvent.
\end{abstract}

Key words: microscopic theory, solvent-solute interaction, hydrodynamic limit, exclusive effect, momentum conservation, generalized Langevin equation

PACS: $66.10 .-x, 05.20 . J j, 05.60 . C d, 87.15 . V v, 47.10 .-g$

\section{Introduction}

When a solute particle diffuses in a solution, a spatial distribution of solvent particles around the solute can largely affect the diffusion. Solvent particles distribute around the solute even when the solute does not move in the equilibrium state. A high density of solvent particles around the solute causes a decrease in a value of the diffusion coefficient. The distribution is directly affected by interaction between solute and solvent particles. Thus, by observing diffusion of the solute in the solvent, one can study solute-solvent interaction if the relationship between the interaction and diffusion is established.

Such effects of a solvent distribution have been observed by recent experiments on diffusion of proteins [1-3]. In the experiments, diffusion of Cytochrome $c$ in water has been observed using the transient grating technique [1,2]. Diffusion of Cytochrome $c$ was three times slower after the formation of hydrogen bonds [1]. A distribution of solvent particles changed when the hydrogen bonds formed. One can expect this change to have caused a decrease in the value of the diffusion coefficient.

When a solute particle is large, a hydrodynamic effect is also important. Motions of a solute cause motions of solvent particles by collisions. Solvent particles moved by the solute collide with a solute, again. The collision affects diffusion of the solute. The effect turns out to be long ranged because the momentum of solvent and solute particles is conserved.

The hydrodynamic effect is described by the Stokes law, which can be derived by applying the Stokes approximation to the hydrodynamic equations. The Stokes law can be derived from the Navier-Stokes equation including viscosity [4]. The approximation is given by the neglect of nonlinear advection terms.

The diffusion coefficient calculated using the Stokes law depends only on the size of a solute particle [4]. The expression does not include the other parameters of a solute. This shows that the hydrodynamic effect depends little on the particularities of interaction between solvent and solute particles.

The hydrodynamic effect has been shown to be dominant when a solute is ten times as large as a solvent particle, by recent simulations [5]. By the simulations, the diffusion coefficients were

*E-mail: yosi3scp@mbox.nc.kyushu-u.ac.jp 
calculated in the systems of hard spheres with various size ratios between the solute and solvent. When the ratio was larger than about 10, the Stokes law was valid. A dominant hydrodynamic effect is shown by the validity of the Stokes law, because the other effects are not considered by the Stokes law.

The simulation results contrast with the experimental results. The size ratio of 10 employed by the simulation is similar to the ratio of Cytochrome $c$ to water. The simulations with the ratio showed the validity of the Stokes law, which is not affected by a distribution of solvent particles. Thus, the experimental results contrast with the validity of the Stokes law because the hydrogen boding is important in the experiments.

Comparison of the simulations with the experiments on Cytochrome $c$ clarifies the importance of attractive interaction between solute and solvent particles. Attractive interaction, such as hydrogen boding, was not considered by the simulations. The systems employed by the simulations consisted of hard spheres. In the experiments, however, attractive interaction plays an important role. Thus, one can expect that attractive interaction brings in experimental results in contrast to the results obtained by simulations.

To study the effects of attractive interaction theoretically, one needs a molecular theory giving the correct limit of a large solute. Whenever one studies diffusion of a large solute theoretically, the theories should have an exact asymptotic expression in the limit of a large solute. If the exact limit is not given, the diffusion coefficient calculated by the theory deviates from the exact value when a solute is large. The exact limit of a large solute is given by the diffusion coefficient proportional to $1 / R$ where $R$ is the radius of the solute. Apart from the correct limit, in order to study the effects of attractive interaction, one needs to explicitly deal with the interaction between solute and solvent particles.

A molecular theory of this kind turns out to be the mode coupling theory [6,7]. The mode coupling theory has been widely employed when calculating the dynamical properties of liquids such as a diffusion coefficient [8-11]. The mode coupling theory gives the correct limit of a large solute by the transverse coupling term [7]. Using the theory, one can consider a solvent distribution through the radial distribution function and the direct correlation function between the solute and solvent.

The mode coupling theory, however, cannot adequately deal with the effects of a solute on motions of solvent particles. In the mode coupling theory, motions of solvent particles are included by the dynamic structure factor and transverse current autocorrelation function in a bulk solvent [7]. They do not have the effects of a solute. One cannot neglect the effects of a solute on motions of solvent particles, when the interaction between solute and solvent particles is strong.

To deal with the effects of a solute on motions of solvent particles, Yamaguchi et al. have developed a new molecular theory [12]. The theory was formulated using the generalized Langevin equation [13] in an inhomogeneous system. They considered an inhomogeneous system, regarding the interaction between solute and solvent particles as an external field.

The theory developed by Yamaguchi et al. [12] can be obtained using the time dependent density functional method (TDDFM) [14-18]. One can derive the theory by Yamaguchi et al. [12] from the TDDFM, adding the memory effects and momentum conservation. In the same way as the theory by Yamaguchi et al. [12], the TDDFM adequately deals with the attractive interaction. The equation of the TDDFM, however, does not include the terms of the memory effect. In addition, when the TDDFM is calculated, momentum does not conserve.

Using the TDDFM, the diffusion coefficients have been calculated [19-21]. The calculations did not agree with molecular dynamics simulations [19]. The disagreement was caused by the neglect of the momentum conservation. The basic equation of the TDDFM does not have the terms of memory effects as well. The diffusion coefficient, however, is not affected by the memory terms because one can calculate the diffusion coefficient only in case of obtaining the density field in the steady state.

Though the theory by Yamaguchi et al. [12] adequately deals with the solute effect on solvent motions, the correct limit of a large solute has never been proved so far. In the limit of a large solute, the diffusion coefficient should be proportional to $1 / R$. By their theory, Yamaguchi et al. 
have numerically studied the diffusion of a large solute on their own [22]. These numerical studies showed the dependence of $1 / R$ when the ratio between the solute and solvent radii was 8 . The infinite limit, however, has never been exactly studied.

On the other hand, when considering diffusion of a large solute, one has a problem of the boundary condition. The boundary condition on the surface of the solute should be imposed to calculate the diffusion coefficient using the hydrodynamic equations. One usually assumes the stick or slip boundary condition. Whether the stick or slip boundary condition is assumed, one can obtain the dependence of $1 / R$, though the proportional coefficient is different.

None has clearly understood the relationship between the boundary condition and molecular theories. Though the boundary condition should be assumed in the hydrodynamic equations, they are not needed by molecular theories. This is because the molecular theories explicitly deal with interaction between the solute and solvent particles. On the other hand, molecular theories should agree with the hydrodynamic equations in the limit of a large solute. Thus, the boundary conditions can be derived from molecular theories by the limit of a large solute.

The theory developed by Yamaguchi et al. [12] turns out to be adequate to the study of the boundary conditions because the hydrodynamic equations can be derived from their theory. Using the molecular theory by Yamaguchi et al. [12], one can derive the hydrodynamic equations with the boundary condition in the system of solute and solvent particles. Since the boundary condition is expressed by molecular parameters, one can understand the boundary condition at the molecular level.

The derivation of the hydrodynamic equations from the theory by Yamaguchi et al. [12] is easy because the basic equations are the same types as the hydrodynamics. They are the continuum equation for the density field and the equation of motion for the current density field. The equations of motion agree with the momentum conservation.

When the hydrodynamic equation is derived, the molecular theory by Yamaguchi et al. [12] contrasts with the mode coupling theory. Using the mode coupling theory, one can directly calculate the diffusion coefficient without equations of motion. The hydrodynamic equations cannot be derived from the mode coupling theory.

The purpose of the present study is to obtain the exact large-solute limit in the theory developed by Yamaguchi et al. [12]. In addition, the boundary condition is derived at the molecular level. The system consists of a spherical solute particle dissolved in spherical solvent particles. The limit of a large solute in the theory is analytically obtained in the system. The large-solute limit gives the boundary condition on the surface of the solute without any other assumptions.

\section{A generalized Langevin equation theory in an inhomogeneous system}

In the present study, a solute is fixed in a flowing solvent. The solvent particles have the velocity $\mathbf{u}$ at an infinite distance from the solute. The present system is equivalent to a system of a solute moving with the velocity of $-\mathbf{u}$. Solute and solvent particles are simple liquids with spherical interaction. One can obtain the diffusion coefficient, calculating the density field for the flowing solvent particles in a steady state.

The steady-state density field of the flowing solvent particles is given using the theory developed by Yamaguchi et al. [12] by

$$
\begin{aligned}
& \nabla \cdot \mathbf{J}(\mathbf{r})=0, \\
& \rho_{\text {eq }}(\mathbf{r}) \nabla \frac{P(\mathbf{r})}{\rho_{\text {eq }}(\mathbf{r})}+\int \mathrm{d} \mathbf{r}^{\prime} \mathbf{M}\left(\mathbf{r}-\mathbf{r}^{\prime}\right) \frac{\mathbf{J}\left(\mathbf{r}^{\prime}\right)}{\rho_{\mathrm{eq}}\left(\mathbf{r}^{\prime}\right)}=0,
\end{aligned}
$$

where

$$
P(\mathbf{r})=k_{\mathrm{B}} T \delta \rho(\mathbf{r})-k_{\mathrm{B}} T \rho_{\mathrm{eq}}(\mathbf{r}) \int \mathrm{d} \mathbf{r}^{\prime} c\left(\mathbf{r}-\mathbf{r}^{\prime}\right) \delta \rho\left(\mathbf{r}^{\prime}\right) .
$$

Here, $\mathbf{J}(\mathbf{r})$ is the current density field at the steady state. It satisfies the boundary condition: $\mathbf{J}(\mathbf{r}) \rightarrow \rho \mathbf{u}$ at $r \rightarrow \infty$, where $\rho$ is the density of the solvent and $r$ is the distance from the solute. 
In equations $(2)$ and $(3), \rho_{\text {eq }}(\mathbf{r})$ is the inhomogeneous equilibrium density field $(u=0)$. It depends explicitly on $r$ because the solute affects the density field of the solvent. In equation (3), $\delta \rho(\mathbf{r})=\rho(\mathbf{r})-\rho_{\text {eq }}(\mathbf{r})$ where $\rho(\mathbf{r})$ is the density field at the nonequilibrium steady state.

In addition, $\mathbf{M}(\mathbf{r})$ is the tensor obtained by a random force, and $c(\mathbf{r})$ is the direct correlation function of solvent particles. One can obtain $\mathbf{M}(\mathbf{r})$, integrating the time correlation function of a random force from $t=0$ to $\infty$. The tensor $\mathbf{M}(\mathbf{r})$ and the direct correlation function $c(\mathbf{r})$ are approximately given in the homogeneous system without the solute. Then, $\mathbf{M}(\mathbf{r})$ is explicitly expressed by [12]

$$
\int \mathbf{M}(\mathbf{r}) \mathrm{e}^{\mathrm{i} \mathbf{k r}} \mathrm{d} \mathbf{r}=\mathbf{k} \otimes \mathbf{k} \tilde{K}_{L}(k)+\left(k^{2} \mathbf{U}-\mathbf{k} \otimes \mathbf{k}\right) \tilde{K}_{T}(k),
$$

where $k=|\mathbf{k}|$ and $\mathbf{U}$ is the unit tensor. Here, $\tilde{K}_{L}(k)$ and $\tilde{K}_{T}(k)$ are the longitudinal and transverse parts of the time integral for the correlation function of a random force [12]. They are related to the dynamical structure factor and transverse current correlation function.

Equations (1) and (2) are the generalized Langevin equations [13] in an inhomogeneous system. The present inhomogeneity is caused by the solute. The density of solvent particles depends on the distance from the solute.

Equations (1) and (2) describe the inhomogeneous system by the equilibrium density field $\rho_{\text {eq }}(\mathbf{r})$. Because of $\rho_{\text {eq }}(\mathbf{r})$, equation (2) does not have the translational invariant. This contrasts with usual homogeneous generalized Langevin equations extensively employed in the study of the dynamics of liquids [8]. The inclusion of $\rho_{\text {eq }}(\mathbf{r})$ in equation (2) allows one to adequately deal with strong attractive interaction using the theory by Yamaguchi et al. [12].

To derive equations (1) and (2), Yamaguchi et al. [12] had two approximations to the exact framework of the generalized Langevin equations [13]. The first approximation shows that a density fluctuation in the inhomogeneous system is given at the level of the hyper-netted chain theory [8]. From this approximation, one can calculate the direct correlation function $c(\mathbf{r})$ by a homogeneous system without the solute. The second approximation is given by the random force in a homogeneous system. Thus, $\mathbf{M}(\mathbf{r})$ is given by equation (4).

The theory developed by Yamaguchi et al. [12] is applied to the present solute-solvent system with assumptions on the inside of the solute. The assumption is given by $\rho_{\text {eq }}(\mathbf{r})=0$ for $r \leqslant R$, where $R$ is the radius of the solute. In addition,

$$
f(r) \equiv \frac{1}{\rho_{\mathrm{eq}}(\mathbf{r})} \frac{\mathrm{d} \rho_{\mathrm{eq}}(\mathbf{r})}{\mathrm{d} r} \rightarrow \infty
$$

is also assumed at $r \leqslant R$. These are assumed because interaction between the solvent and solute particles diverges in all regions of $r \leqslant R$.

Besides the assumptions about the inside, equations (1) and (2) are assumed to be valid in the whole space of the system. The validity on the inside of the solute, in particular, is assumed. In addition, $\mathbf{M}\left(\mathbf{r}-\mathbf{r}^{\prime}\right)$ is assumed to be a smooth function. Then, the value of $\mathbf{M}\left(\mathbf{r}-\mathbf{r}^{\prime}\right)$ is not zero even if a value of $\left|\mathbf{r}-\mathbf{r}^{\prime}\right|$ is large.

From the assumptions, one can show that the velocity field $\mathbf{v}(\mathbf{r})$ defined by $\mathbf{J}(\mathbf{r}) / \rho_{\text {eq }}(\mathbf{r})$ does not diverge on the inside of the solute $r<R$. If $\mathbf{v}(\mathbf{r})$ diverges for $r<R$, the second term on the left hand side of equation (2) diverges in all regions. This is because $\mathbf{M}\left(\mathbf{r}-\mathbf{r}^{\prime}\right)$ has a small value even for a large value of $\left|\mathbf{r}-\mathbf{r}^{\prime}\right|$. Then, equation (2) shows that the first term also diverges in all regions. Thus, $\mathbf{v}(\mathbf{r})$ does not diverge for $r<R$ so that the first term in equation (2) does not diverge for $r>R$.

By the definition of the velocity field, one finds that the components except the radial direction can have a non-zero value on the inside of the solute. On the inside of the solute, the current density field $\mathbf{J}(\mathbf{r})$ and density field $\rho_{\text {eq }}(\mathbf{r})$ vanish. Since the velocity field $\mathbf{v}(\mathbf{r})$, however, is defined by the ratio of $\mathbf{J}(\mathbf{r})$ to $\rho_{\mathrm{eq}}(\mathbf{r})$, it can have a value that is not zero.

On the other hand, the velocity field of the radial direction should vanish on the inside of the solute so that the velocity field does not diverge on the inside. If the velocity field of the radial direction is denoted by $v_{\mathrm{r}}(\mathbf{r})$, using $f(r)$ in equation (5), one can rewrite equation (1) by

$$
f(r) v_{\mathrm{r}}(\mathbf{r})=-\nabla \cdot \mathbf{v}(\mathbf{r})
$$


Since $\mathbf{v}(\mathbf{r})$ does not diverge on the inside of the solute, equation (6) leads to

$$
v_{\mathrm{r}}(\mathbf{r})=0
$$

for $r<R$.

In addition, the pressure $P(\mathbf{r})$ in equation (2) should vanish on the inside of the solute. Equation $(2)$ is written in the form

$$
-\hat{\mathbf{r}} f(r) P(\mathbf{r})+\nabla P(\mathbf{r})+\int \mathrm{d} \mathbf{r}^{\prime} \mathbf{M}\left(\mathbf{r}-\mathbf{r}^{\prime}\right) \mathbf{v}\left(\mathbf{r}^{\prime}\right)=0
$$

where $\hat{\mathbf{r}}$ is the unit vector of the radial direction. The first term diverges with $f(r)$, though the third term $\int \mathrm{d} \mathbf{r}^{\prime} \mathbf{M}\left(\mathbf{r}-\mathbf{r}^{\prime}\right) \mathbf{v}\left(\mathbf{r}^{\prime}\right)$ does not diverge. Thus, to satisfy equation (8), one has

$$
-f(r) P(\mathbf{r})+\frac{\partial P(\mathbf{r})}{\partial r}=0
$$

Solving the equation, one can obtain $P(\mathbf{r}) \propto \rho_{\text {eq }}(\mathbf{r})$ and $P(\mathbf{r})=0$ for $r<R$.

\section{Limits of a large solute in the molecular theory}

The present section shows the limit of a large solute in the molecular theory introduced in the previous section. In the previous section, equations (1) and (2) were presented to study the steady state using the theory developed by Yamaguchi et al. [12]. In the present section, the limit of a large solute in equations (1) and (2) is given by $R \rightarrow \infty$ with $\overline{\mathbf{r}} \equiv \mathbf{r} / R$ fixed when $\mathbf{v}(\mathbf{r})=\overline{\mathbf{v}}(\overline{\mathbf{r}})$ and $P(\mathbf{r})=\bar{P}(\overline{\mathbf{r}}) / R$ are assumed.

The term of integration in equation (2) is first calculated in the limit of a large solute. The term in equation (2) is written in the form

$$
\int \mathrm{d} \mathbf{r}^{\prime} \mathbf{M}\left(\mathbf{r}-\mathbf{r}^{\prime}\right) \frac{\mathbf{J}\left(\mathbf{r}^{\prime}\right)}{\rho_{\mathrm{eq}}\left(\mathbf{r}^{\prime}\right)}=\int \mathrm{d} \mathbf{r}^{\prime} \mathbf{M}\left(\mathbf{r}^{\prime}\right) \overline{\mathbf{v}}\left(\overline{\mathbf{r}}-\frac{\mathbf{r}^{\prime}}{R}\right) .
$$

In the limit of $R \rightarrow \infty$, from equation (4), one has

$$
\lim _{R \rightarrow \infty} R^{2} \int \mathrm{d} \mathbf{r}^{\prime} \mathbf{M}\left(\mathbf{r}^{\prime}\right) \overline{\mathbf{v}}\left(\overline{\mathbf{r}}-\frac{\mathbf{r}^{\prime}}{R}\right)=-\eta \bar{\nabla}^{2} \overline{\mathbf{v}}(\overline{\mathbf{r}})-\gamma \bar{\nabla}\{\bar{\nabla} \cdot \overline{\mathbf{v}}(\overline{\mathbf{r}})\},
$$

where $\bar{\nabla}$ denotes the gradient of $\overline{\mathbf{r}}$ and

$$
\begin{aligned}
\eta & =\tilde{K}_{T}(0) \\
\gamma & =\tilde{K}_{L}(0)-\tilde{K}_{T}(0)=\zeta+\frac{\eta}{3}
\end{aligned}
$$

Here, $\eta$ and $\zeta$ are the shear and bulk viscosity. The substitution of equation (11) into equation (2) yields

$$
\rho_{\text {eq }}(\mathbf{r}) \bar{\nabla} \frac{\bar{P}(\overline{\mathbf{r}})}{\rho_{\text {eq }}(\mathbf{r})}-\eta \bar{\nabla}^{2} \overline{\mathbf{v}}(\overline{\mathbf{r}})-\gamma \bar{\nabla}\{\bar{\nabla} \cdot \overline{\mathbf{v}}(\overline{\mathbf{r}})\}=0 .
$$

Using the limit in the integration term, one can obtain the continuity of the velocity field on the surface of the solute. Equation (14) is the second differential equation of $\mathbf{v}(\mathbf{r})$. Thus, if the equations are valid in all regions, $\mathbf{v}(\mathbf{r})$ and its first derivative should continue even on the surface of the solute. The first derivative of the radial component $v_{\mathrm{r}}(\mathbf{r})$, however, can be discontinuous because $P(\mathbf{r})$ in equation (14) can be also discontinuous.

One can explicitly express the continuity of the velocity field by equations. To express the equations, the polar coordinate is introduced where the origin is the center of the solute with the 
$z$-axis parallel to the direction of $\mathbf{u}$. Using the coordinate, one has

$$
\begin{aligned}
\lim _{r \rightarrow+R} v_{\mathrm{r}}(\mathbf{r}) & =\lim _{r \rightarrow-R} v_{\mathrm{r}}(\mathbf{r})=0, \\
\lim _{r \rightarrow+R} v_{\theta}(\mathbf{r}) & =\lim _{r \rightarrow-R} v_{\theta}(\mathbf{r}), \\
\lim _{r \rightarrow+R} \frac{\partial v_{\theta}(\mathbf{r})}{\partial r} & =\lim _{r \rightarrow-R} \frac{\partial v_{\theta}(\mathbf{r})}{\partial r}, \\
\lim _{r \rightarrow+R} v_{\psi}(\mathbf{r}) & =\lim _{r \rightarrow-R} v_{\psi}(\mathbf{r}), \\
\lim _{r \rightarrow+R} \frac{\partial v_{\psi}(\mathbf{r})}{\partial r} & =\lim _{r \rightarrow-R} \frac{\partial v_{\psi}(\mathbf{r})}{\partial r},
\end{aligned}
$$

where $v_{\mathrm{r}}(\mathbf{r}), v_{\theta}(\mathbf{r})$ and $v_{\psi}(\mathbf{r})$ are the $r, \theta$, and $\psi$-components of the velocity field. The limits of $r \rightarrow+R$ and $r \rightarrow-R$ denote the approach from the outside and inside of the solute to the surface. Equation (15) was obtained from equation (7).

The next task for obtaining the limit of a large solute in equations (1) and (2) is to calculate the limit in the equilibrium density $\rho_{\text {eq }}(\mathbf{r})$. It is written in the form $\rho_{\text {eq }}(\mathbf{r})=\bar{\rho}(\{r-R\} / \sigma)=$ $\bar{\rho}(\{\bar{r}-1\} R / \sigma)$ where $\sigma$ is the radius of the solvent particle. Since $\rho_{\text {eq }}(\mathbf{r})=\rho_{0}$ for $r \rightarrow \infty$ and $\rho_{\mathrm{eq}}(\mathbf{r})=0$ for $r<R, \rho_{\mathrm{eq}}(\mathbf{r})$ is the step function given by

$$
\lim _{R \rightarrow \infty} \rho_{\mathrm{eq}}(\mathbf{r})=\rho_{0} H(\bar{r}-1) \equiv \begin{cases}\rho_{0} & \bar{r}>1 \\ 0 & \bar{r}<1\end{cases}
$$

where $\rho_{0}$ is the homogeneous density and $\bar{r}=|\overline{\mathbf{r}}|$.

In the large-solute limit, equations (1) and (2) agree with the hydrodynamic equations derived using the Stokes approximation on the outside of the solute. For $r>R$ or $\bar{r}>1$, from equation (20), one has $\rho_{\text {eq }}(\mathbf{r})=\rho_{0}$. Thus, equations (1) and (14) are rewritten by

$$
\begin{aligned}
& \nabla \cdot \mathbf{v}(\mathbf{r})=0, \\
& \nabla P(\mathbf{r})-\eta \nabla^{2} \mathbf{v}(\mathbf{r})=0 .
\end{aligned}
$$

Here, $\bar{r}=r / R, \overline{\mathbf{v}}(\overline{\mathbf{r}})=\mathbf{v}(\mathbf{r})$, and $\bar{P}(\overline{\mathbf{r}})=P(\mathbf{r}) R$ were employed. These are exactly the same equations as the equations by the Stokes approximation.

On the other hand, one can also obtain the equations on the inside of the solute. Since $P(\mathbf{r})=0$ for $r<R$, one can obtain

$$
\hat{\mathbf{r}} Q(\mathbf{r})-\eta \nabla^{2} \mathbf{v}(\mathbf{r})-\gamma \nabla\{\nabla \cdot \mathbf{v}(\mathbf{r})\}=0
$$

Here, $\hat{\mathbf{r}}$ is the unit vector of the radius direction, and $Q(\mathbf{r}) \equiv f(r) P(\mathbf{r})$ can have a finite value because $f(r) \rightarrow \infty$ for $r<R$ though $P(\mathbf{r})=0$. Besides equation (23), one has equation (7) on the inside of the solute. By these equations, one can obtain the velocity field $\mathbf{v}(\mathbf{r})$ on the inside of the solute. The inside of the solute is connected with the outside using equations (15)-(19).

The equations on the inside of the solute can be solved by consideration of the symmetry. From the symmetry, one finds that only $\mathbf{u}$ and $\mathbf{r}$ are vectors given by the present system. Since $\mathbf{v}(\mathbf{r})$ is a vector, $\mathbf{v}(\mathbf{r})$ should be expressed by a linear combination of $\mathbf{u}$ and $\mathbf{r}$. In addition, $\mathbf{v}(\mathbf{r})$ should be a linear function of $\mathbf{u}$. To satisfy these conditions, one has

$$
\mathbf{v}(\mathbf{r})=g(r)(\mathbf{r} \cdot \mathbf{u}) \mathbf{r}+h(r) \mathbf{u},
$$

where $g(r)$ and $h(r)$ are functions of only $r$.

One can explicitly obtain an analytical solution of the equations on the inside of the solute. Since equation $(7)$ gives $(\mathbf{r} \cdot \mathbf{v}(\mathbf{r}))=0$, one has $h(r)=-r^{2} g(r)$. To calculate $g(r)$, the tangental component of equation (23) is calculated by

$$
-\eta\left(\mathbf{e}_{\theta} \cdot \nabla^{2} \mathbf{v}(\mathbf{r})\right)-\gamma\left(\mathbf{e}_{\theta} \cdot \nabla\{\nabla \cdot \mathbf{v}(\mathbf{r})\}\right)=0,
$$


where $\mathbf{e}_{\theta}$ is the unit vector of the $\theta$-direction. The substitution of equation (24) into equation (25) yields $g(r)=c r^{\alpha-2}$, where $c$ is a constant independent of $r$,

$$
\alpha=\frac{-1+\sqrt{9+8 \bar{\gamma}}}{2}
$$

and $\bar{\gamma}=\gamma / \eta$.

The solution on the inside of the solute leads to the boundary condition for the hydrodynamic equations with the Stokes approximation. From equation $(24)$ with $g(r)=c r^{\alpha-2}$ and $h(r)=-c r^{\alpha}$, one has

$$
\frac{\partial v_{\theta}}{\partial r}=\frac{\alpha}{r} v_{\theta}
$$

for $r<R$. From equations (16) and (17), one can obtain

$$
\frac{\partial v_{\theta}}{\partial r}=\frac{\alpha}{R} v_{\theta}
$$

on $r=R$.

The obtained boundary condition can be different from the stick or slip condition, though it can agree with one of them. The stick boundary condition is obtained by equation (28) when $\alpha \rightarrow \infty$. In addition, the slip condition is obtained when $\alpha=1$. The present boundary condition, however, does not agree with that assumed in the Stokes law when $1<\alpha<\infty$.

The present boundary condition is independent of solute properties. The boundary condition in equation (28) depends only on the value of $\alpha$, which is given only by $\eta$ and $\gamma$, as shown by equation (26). The boundary condition, however, should depend on the state of the solute surface from the hydrodynamic viewpoint. This point will be discussed in section 4 .

\section{Discussion}

The present study has shown the limit of a large solute in the molecular theory developed by Yamaguchi et al. [12], on the inside and outside of the solute. On the outside of the solute, in the large-solute limit, the theory has agreed with the hydrodynamic equation derived by the Stokes approximation. One finds that their theory has a correct limit of a large solute in the diffusion coefficient, by the agreement. Thus, their theory can be employed to study diffusion of a large particle. On the other hand, on the inside of the solute, one can obtain an analytical solution, which leads to the boundary condition on the surface of the solute.

On the outside of the solute, the theory developed by Yamaguchi et al. [12] has agreed with the hydrodynamic equation derived by the Stokes approximation. Thus, the calculation by the theory gives the diffusion coefficient proportional to $1 / R$ in the limit of a large solute. The asymptotic form is independent of the boundary condition on the surface of the solute. In spite of the stick, slip, and other boundary conditions, the same asymptotic form is obtained.

The agreement with the Stokes approximation allows one to employ the theory by Yamaguchi et al. [12] to study diffusion of a large solute. To study diffusion of a large solute, one needs a theory that is exact as $R \rightarrow \infty$. The theory by Yamaguchi et al. [12] is satisfied with the condition.

On the other hand, on the inside of the solute, an analytical solution has been obtained using equations (7) and (9) showing that $v_{\mathrm{r}}(\mathbf{r})$ and $P(\mathbf{r})$ vanish on the inside. The limit of a large solute does not affect the validity of the equations (7) and (9). Thus, the solution can be obtained even when equation (20) is not valid. The limit of $M(\mathbf{r})$ in equation (2), however, is required.

The analytical solution on the inside has led to some kinds of boundary conditions. They include not only the stick and slip boundary conditions but also other ones. The boundary conditions depend on the value of $\alpha$ in equation (26). The value is given by the property of the solvent. The boundary condition is not stick or slip if the value of $\alpha$ is not the unity or infinity.

When the diffusion coefficient is proportional to $1 / R$ in the limit of a large solute, these boundary conditions affect the proportional coefficient. Denoting the proportional coefficient by $a$, one 
can express the asymptotic form of the diffusion coefficient by $a k_{\mathrm{B}} T /(\pi \eta R)$. The slip and stick boundary conditions have $a=1 / 4$ and $1 / 6$.

The boundary condition different from the slip or stick condition has also been obtained in the previous studies. The proportional coefficient $a=1 / 5$ was obtained using the mode coupling theory of hard spheres [6]. That is, the boundary condition was different from the slip or stick condition because the value of $a$ was not $a=1 / 4$ or $1 / 6$. In addition, Yamaguchi et al. have numerically calculated the diffusion coefficient using the theory of their own [22]. The calculated diffusion coefficient deviated from that by the slip and stick boundary conditions.

The present boundary condition is independent of the solute property because the limiting theory of a large solute does not include any parameters of the solute except $R$. The property of the solute is presented by $\rho_{\text {eq }}(\mathbf{r})$ in the theory developed by Yamaguchi et al. [12]. When a solute is large, $\rho_{\text {eq }}(\mathbf{r})$ is approximately the step function given by equation $(20)$. It does not include the properties of the solute except $R$, which represents the position of the boundary. This is because the solute size does not affect the range of interaction between solute and solvent particles. Thus, the ratio of the range to $R$ approaches zero as $R \rightarrow \infty$.

The present result of the boundary condition contrasts with the hydrodynamic viewpoint. From the hydrodynamic viewpoint, the boundary condition depends on the state of the solute surface. The state of the solute surface is often expressed by the strength of friction on the surface of the solute.

From the hydrodynamic viewpoint, the stick or slip boundary condition is determined by friction on the surface of the solute. When friction is strong, the boundary condition approaches the stick condition. The slip condition is caused by weak friction. Friction of the solute surface depends on the interaction between solute and solvent particles at the molecular level.

One can study the effects of the solute on the boundary condition by the perturbative expansion from the limit of a large solute. If $R \rightarrow \infty$, the boundary conditions are independent of the solute property. Thus, the boundary conditions can depend on the properties of the solute when $R$ has a finite value. One can study the effects of the finite value of $R$ if the basic equations are expanded in terms of $1 / R$. The boundary condition depends on the properties of the solute from the terms of the first order.

In addition, one can also study the diffusion of proteins by the perturbative expansion in $1 / R$. Since the present theory is formulated at $R \rightarrow \infty$, one cannot consider the effects of the solvent distribution. The perturbative expansion allows one to consider the effects. One cannot calculate the diffusion coefficient of a protein unless the effects are considered, because the experiments showed the importance of the effects.

Numerical studies of the theory obtained by the perturbative expansion are a future work. The numerical studies are interesting because the calculated results can be compared with experiments on diffusion of proteins. In addition, the numerical calculations are easier than the original theory developed by Yamaguchi et al. [12]. The perturbative expansion theory requires studies on the limit of $R \rightarrow \infty$. Thus, the present study is a first step in the development of the perturbative expansion theory.

\section{Acknowledgements}

I wish to thank Professor T. Yamaguchi for helpful comments. This work was supported by the Grant-in-Aid for Scientific Research on Priority Area and for Scientific Research (c) from the Japanese Ministry of Education, Science, Sports, and Culture.

\section{References}

1. Nishida S., Nada T., Terashima M., Biophys. J., 2004, 87, 2663.

2. Nishida S., Nada T., Terashima M., Biophys. J., 2005, 89, 2004.

3. Khan J.S., Imamoto Y., Harigai M., Kataoka M., Terashima M., Biophys. J., 2006, 90, 3686.

4. Landau L.D., Lifshitz E.M. Fluid Mechanics. Butterworth-Heinemann, Oxford, 1987. 
5. Sokolovskii R.O., Thachuk M., Patey G.N., J. Chem. Phys., 2006, 125, 204502.

6. Mehafty J.R., Cukier R.I., Phys. Rev. Letters, 1977, 38, 1039.

7. Bhattacharyya S., Bagchi B., J. Chem. Phys., 1997, 106, 1757.

8. Hansen J.P., MacDonald I.R. Theory of Simple Liquids. Academic press, London, 1986.

9. Sjögren L., Sjölander A., J. Phys. C, 1979, 12, 4369.

10. Kirkpatrick T.R., Nieuwoudt J.C., Phys. Rev. A, 1986, 33, 2651.

11. Kirkpatrick T.R., Nieuwoudt J.C., Phys.Rev. A, 1986, 33, 2658.

12. Yamaguchi T., Matsuoka T., Koda S., J. Chem. Phys., 2005, 123, 034504.

13. Mori H., Prog. Theor. Phys., 1965, 33, 423.

14. Yoshimori A., Journal of Theoretical and Computational Chemistry, 2004, 3, 117.

15. Yoshimori A., Phys. Rev. E, 2005, 71, 031203.

16. Chandra A., Bagchi B., J. Chem. Phys., 1989, 91, 1829.

17. Munakata T., J. Phys. Soc. Jpn., 1990, 59, 1299.

18. Marconi U.M.B., Tarazona P., J. Chem. Phys., 1999, 110, 8032.

19. Araki J., Munakata T., Phys. Rev. E, 1995, 52, 2577.

20. Yoshida S., Hirata F., Munakata T., Phys. Rev. E, 1996, 54, 1763.

21. Yoshimori A., Day T.J.F., Patey G.N., J. Chem. Phys., 1998, 108, 6378.

22. Yamaguchi T., Matsuoka T., Koda S., J. Mol. Liq., 2007, 134, 1.

\title{
Молекулярна теорія дифузії великих частинок в розчині
}

\author{
А.Йошіморі \\ Фізичний факультет, Кюшу університет, Фукуока, Японія 812-8581 \\ Отримано 4 вересня 2007 р., в остаточному вигляді - 11 жовтня 2007 р. \\ Вивчається границя великої розчинюваної частинки в рамках молекулярної теорії, розвинутої Яма- \\ гучі. В цій границі наближення Стокса до гідродинамічних рівнянь отримано за межами дифузії \\ розчинюваної частинки. Границя великої частинки також приводить до рівнянь всередині області \\ розчинюваної частинки. Аналітичний розв'язок дозволяє отримати граничні умови на поверхні ча- \\ стинки, необхідні при розрахунку гідродинамічних рівнянь. Граничні умови в спеціальному випадку \\ включають граничні умови зміщення, що використовуються в законі Стокса. Крім цих, можуть бути \\ отримані також інші умови. Граничні умови залежать від властивостей розчинника.
}

Ключові слова: мікроскопічна теорія, взаємодія розчинник-розчинювана речовина, гідродинамічна границя, ексклюзивний ефект, момент збереження, узагальнене рівняння Ланжевена

PACS: $66.10 .-x, 05.20 . J j, 05.60 . C d, 87.15 . V v, 47.10 .-g$ 
